\title{
Consciencia, Cientificismo y Multideterminismo en Psicoanálisis
}

RESUMEN : Se examinan los conceptos de lo consciente y lo inconsciente, objetividad y subjetividad, así como la dimensión científica del psicoanálisis, sus limitaciones y su multideterminismo.

PALABRAS CLAVE : psicoanálisis, consciencia, transferencia, multideterminismo.
SUMMARY : The concepts of conscious and unconscious, objectivity and subjectivity, and so the scientific dimension of psychoanalysis are examined, together with its limitations and multideterminism. KEY WORDS: psychoanalysis, consciousness, transference, multideterminism.

\section{Lo consciente y lo inconsciente}

En la actualidad existe una explosión en la investigación neurocientífica y psicológica cognitiva de los procesos inconscientes, tras largos años de desinterés e incluso incredulidad en su existencia y en el papel que podían desempeñar en la percepción y la memoria (1).

La mayoría de los estímulos externos y de procedencia interna que influyen sobre el estado de ánimo y el comportamiento del ser humano no son percibidos conscientemente por éste. La consciencia es una cualidad psíquica especial del conocimiento que supone un nivel superior de percepción en cuanto a organización mental. Constituye una experiencia subjetiva de recepción éxtero e interoceptiva que prepara al individuo para las respuestas inteligentes a los estímulos. Freud (2) comparó la consciencia a un órgano sensorial para la percepción de las cualidades psíquicas. En efecto, percibe la calidad (placentera o displacentera) de las sensaciones, los impulsos, los recuerdos, las fantasías y los razonamientos en un contexto temporal y espacial (3). También, en virtud de su función perceptiva de los estímulos externos a través de los órganos de los sentidos, la consciencia constituye la base para la comprobación de la realidad.

En la actualidad, los psiquiatras y los psicólogos de cualquier orientación teórica reconocen que gran parte de la vida mental se procesa inconscientemente (fenómeno que puede captarse en neuroimagen). En los últimos años se han descubierto, por ejemplo, localizaciones específicas cerebrales para el procesamiento inconsciente de las percepciones visuales. Hemos de recordar que hasta que Freud introdujo la idea de una actividad psíquica inconsciente en el mundo científico-médico, este concepto había sido considerado un absurdo. Aunque hubo pensadores anteriores que formularon la existencia de un mundo inconsciente (4), durante siglos, médicos y filósofos habían creído que la actividad psíquica tenía que ser, por definición, accesible a la consciencia. En palabras de Descartes, «la mente es transparente para sí misma». Freud mantuvo todo lo contrario: la actividad mental era de por sí inconsciente; se hacía consciente sólo cuando se dirigía hacia ella cierta forma de atención, en el caso del sistema Preconsciente, o por medio de la introspección activa y la explo- 
DEBATES

ración analítica en el caso del Inconsciente. Desde esta revolucionaria perspectiva, lo que habría debido suscitar mayor interés en los investigadores no era que existieran fenómenos psíquicos inconscientes, sino el fenómeno en sí de la consciencia.

En la llamada «teoría topográfica» o «primera tópica» freudiana, el padre del psicoanálisis distinguió el sistema Preconsciente, del Inconsciente (5). Aunque ambos eran descriptivamente inconscientes, los contenidos del primer sistema resultaban potencialmente accesibles a la consciencia o sistema Consciente, mientras que los del segundo eran, además, dinámicamente inconscientes, esto es, no podían aflorar a la consciencia debido a que resultaban demasiado conflictivos por su naturaleza sexual o agresiva, censurable y ansiógena. Ante estos contenidos se erigía la barrera de la represión. La existencia de mecanismos represores, es decir, de contrafuerzas mentales encargadas de mantener inconscientes de modo selectivo ciertas ideas y afectos, no se basa solamente en las observaciones clínicas de los psicoanalistas; hay numerosos estudios en Psicología Experimental que apoyan la existencia de este fenómeno $(6,7)$. Sin embargo, no es necesario recurrir a ellos para demostrar la existencia de dicho mecanismo. Prueba de éste es la amnesia de tantos sentimientos de la niñez relacionados con la sexualidad y la agresión. Esta amnesia, que casi todos podemos constatar en nuestras vidas, es precisamente lo que hace posible negar la existencia de las pulsiones agresivas y sexuales consustanciales al psiquismo infantil, cuando una sencilla observación no prejuiciada tiene que hacer concluir a cualquiera que los niños tienen evidentemente tendencias agresivas y curiosidad sexual.

Así pues, pocas dudas puede haber acerca de la existencia de un mundo mental inconsciente. A cualquier persona con una mínima inclinación introspectiva le resultará imposible conceptuar al Inconsciente como mera entelequia. Todos recordamos ideas que se nos habían olvidado, podemos descubrir en ciertos recuerdos significados que no discernimos en la situación original, soñamos imágenes que nos parecen «impensables» (i.e., reprimidas), sentimos antipatías y simpatías que no podemos explicar, percibimos en los demás y en nosotros mismos una ceguera selectiva respecto a algunos temas, etc. Por poner un ejemplo común, a todos nos ha sucedido el darnos cuenta de omisiones y distorsiones en nuestros recuerdos al ver una película por segunda vez. El hecho de que una parte de la mente (el Consciente) ignora lo que ocurre en otra parte de ésta resulta clínicamente muy evidente en los estados disociativos. Pero, además, la existencia de contenidos mentales inconscientes puede ser demostrada experimentalmente por medio de la hipnosis. A través de ésta, en efecto, pueden recobrarse recuerdos que el sujeto no creía tener, y puede inducirse amnesia o insensibilidad perceptual selectiva (como en las alucinaciones negativas) (8).

\section{Filogénesis de la consciencia}

Puede argüirse que los animales son también «conscientes» de algunas sensaciones, porque, como los humanos, sienten dolor, temor, hambre, etc. La diferencia estriba en que parecen carecer de consciencia reflexiva. En efecto, el animal, o el niño de pecho, siente, por ejemplo, hambre, pero no tiene concepto de lo que es el hambre. 
DEBATES

Se ha conjeturado mucho acerca del origen y la naturaleza evolutiva de la consciencia. Humphrey (9) formuló la teoría de que existió una presión en la selección de los homínidos para el desarrollo de aquel tipo de psicología que les permitiese leer, por así decir, las intenciones de sus congéneres, usando como modelo una imagen de sí mismos. La capacidad de introspección nos habría proporcionado el mejor conjunto de elementos de juicio para predecir la conducta (amigable u hostil) del prójimo. El procesamiento reflexivo de datos inherente a la comprensión consciente de nuestro comportamiento nos habría permitido una toma de decisiones más adaptativa (10). El cerebro evolucionó como un procesador de información que internalizaba los datos del entorno, haciendo al organismo más receptivo a los estímulos ambientales y más reactivo a ellos. Filogenéticamente, los cerebros primitivos reaccionan de forma refleja. En el caso de los vertebrados superiores, el cerebro fue complicándose porque la evolución favoreció aquellos sistemas nerviosos capaces de responder rápidamente pero también de modo flexible a los estímulos, con el fin de adaptarse a la mayor cantidad posible de cambios en las circunstancias ambientales (11).

La consciencia proporciona los medios que nos permiten percibir los cambios y adaptarnos de un modo más funcional a ellos -aunque se vea tan a menudo interferida por procesos inconscientes. La consciencia implica un sistema de retroalimentación que posibilita no sólo tener en cuenta las modificaciones del entorno sino también las propias respuestas a dichos cambios, tomando a éstos como objetos en sí de atención. Ello abre las puertas a un fenómeno inédito en la evolución de la biología cerebral, característico de nuestra especie: la posibilidad de manejo en la memoria y en la fantasía de las representaciones de uno mismo independientemente de las respuestas a la realidad concreta externa.

En el caso general de los vertebrados superiores con capacidades de funcionamiento cerebral tan alejadas de los programas reflexológicos del sistema nervioso de animales inferiores, los dictados genéticos no son suficientes ni para explicar satisfactoriamente la conducta ni para hacer a ésta inter ni intraespecíficamente adaptativa. La versatilidad en la expresión del genoma (la epigénesis) es el factor esencial en este asunto. Lo adquirido en los años formativos, la comunicación simbólica, el aprendizaje cumulativo y su transmisión (las culturas) separan a los seres llamados racionales de los irracionales. Isócrates, hace veinticuatro siglos, escribió: «En la mayor parte de nuestras capacidades no nos distinguimos en absoluto de los animales; de hecho, somos inferiores a muchos de ellos por lo que respecta a la rapidez, la fuerza y otros recursos. Pero gracias a que se ha desarrollado en nosotros el poder de persuadirnos unos a otros y de mostrarnos a los demás en la forma que deseamos, hemos podido evitar vivir como las bestias» (12).

\section{Objetividad y subjetividad}

Existe un mundo objetivo y uno subjetivo: el mundo del noumenon kantiano inherentemente incognoscible y el mundo fenoménico, es decir, el reino de nuestras percepciones sobre el mundo objetivo, sobre las «cosas en sí mismas», en la terminología del 
idealismo trascendental. Este mundo fenoménico, este mundo de las sombras en la caverna (por usar ahora la famosa alegoría platónica) es lo que puede estudiar la ciencia psicológica. Comentó Pinker (13), «Platón dijo que nos hallamos en una caverna y que conocemos el mundo sólo por las sombras que éste proyecta sobre las paredes. Pues bien, la caverna es nuestro cráneo y las sombras, nuestras representaciones mentales». Freud (5) señaló que nuestras limitaciones a la hora de la aprehensión del mundo objetivo son aplicables también a nuestra comprensión de la vida mental: «La hipótesis psicoanalítica de la actividad psíquica inconsciente constituye [...] una extensión de la rectificación, llevada a cabo por Kant, de la teoría de la percepción externa. Del mismo modo que Kant nos invitó a no desatender la condicionalidad subjetiva de nuestra percepción y a no considerar nuestra percepción idéntica a lo percibido incognoscible, nos invita el psicoanálisis a no confundir la percepción de la conciencia con los procesos psíquicos inconscientes objetos de la misma».

Los elementos objetivos de la naturaleza, en efecto, son percibidos de formas diferentes por distintos seres vivos, dependiendo del grado diferencial de evolución de los órganos sensoriales y de la capacidad integradora del sistema nervioso central. Pensemos por un momento en cómo puede percibir un murciélago nuestro entorno, por no hablar de animales invertebrados. Pero es que las diferencias en la percepción del mundo externo puede variar muy considerablamente también dentro de la misma especie humana; pensemos en el mundo del ciego, del recién nacido, o de la persona psicótica, por ejemplo.

Freud enseñó que la consciencia era el órgano perceptual de nuestra vida mental, que es originalmente inconsciente. La consciencia no sería una mera porción de la actividad psíquica, sino una percepción o una reflexión sobre ésta, de por sí inconsciente (14). En palabras de Freud (2), «Lo inconsciente es lo psíquico verdaderamente real: su naturaleza interna nos es tan desconocida como la realidad del mundo exterior y nos es dado por el testimonio de nuestra conciencia tan incompletamente como el mundo exterior por el de nuestros órganos sensoriales». Esta perspectiva representa un giro copernicano, porque lo que hay que explicar entonces no es la existencia del Inconsciente, sino el hecho de que pueda darse el fenómeno de la consciencia. Con el psicoanálisis se pretende ampliar el campo de ésta y corregir sus distorsiones, capacitándola para conseguir percepciones progresivamente objetivas.

\section{La atención}

Así pues, un paso fundamental para entender la mente humana es el reconocimiento de que existen pensamientos, sentimientos y sensaciones más allá de la vida consciente. El campo de la consciencia es en realidad sorprendentemente limitado: podemos enfocar la atención consciente sólo en una actividad y no en dos a la vez; los inputs perceptuales se interfieren entre sí. No podemos escuchar atentamente la radio y estudiar a la vez-digan lo que digan nuestros adolescentes. Además, la aplicación de un foco consciente (la «catexia de atención» que decimos en psicoanálisis) a una actividad automática preconsciente sue- 
DEBATES

le interferir con el funcionamiento de esta última. Todos sabemos el riesgo que corremos al bajar las escaleras si pensamos deliberadamente en el movimiento de nuestras piernas.

Cuanto más estudiamos la mente humana, mayor cuenta nos damos de lo limitado del campo de la experiencia consciente. Pero, además, resulta que esta misma experiencia (en sueños, lapsus, síntomas, por ejemplo), puede hallarse gobernada por leyes que están más allá de la consciencia (15). «¿Qué misión queda, pues, en nuestra representación, a la conciencia, antes omnipotente y que todo lo encubría?», preguntó Freud (2): «Sencillamente la de un órgano sensorial para la percepción de cualidades psíquicas». Observemos por otra parte que en cualquier investigación sobre la psicología humana, el objeto de estudio es simultáneamente el instrumento para llevar éste a cabo. La consciencia aparece, pues, como un aspecto pequeño de la vida mental, aunque absolutamente esencial en nuestra humanidad. Además, en el decir de Freud (16), «es la única luz que nos guía en las tinieblas de la psicología de las profundidades».

La consciencia es un fenómeno de naturaleza psicológica que nos permite darnos cuenta $o$, valga la redundancia, ser conscientes de nuestros propios pensamientos, nuestros recuerdos, nuestras fantasías, nuestras sensaciones, nuestras acciones motoras, de nuestra existencia y del hecho mismo de ser conscientes. No se sabe con exactitud cuáles son los mecanismos fisiológicos de los fenómenos de la consciencia y la atención. Sabemos, desde luego, que como fundamento físico tienen el funcionamiento cortical, sobre todo del hemisferio izquierdo, y el del sensorium comunis del tálamo, y como substrato químico los sistemas neuromoduladores aminérgico (noradrenérgico y serotoninérgico) y colinérgico (17), pero es mucho lo que aún se desconoce sobre la neurobiología de la atención consciente.

\section{Novedad y transferencia}

Por lo general, las situaciones nuevas resultan potencialmente amenazantes porque nos recuerdan inconscientemente a cuando las vivimos en la indefensión de la infancia. Se comprende entonces que la mente recurra automáticamente a protegerse de la sensación de inseguridad resultante encontrando parecidos tranquilizadores entre lo nuevo y lo conocido. La tendencia del ser humano a hacer casar las nuevas percepciones con lo que ya nos resulta familiar ha sido reconocida desde antaño. El filósofo del siglo dieciocho, Gianbattista Vico, escribió: «Es una propiedad de la mente que cuando los hombres no pueden formarse una idea de cosas distantes y desconocidas, las juzgan por lo que tienen cerca y conocen. Este axioma muestra cuál es la fuente inextinguible de todos los errores sobre los principios de humanidad que han sido adoptados por naciones enteras y por todos los eruditos" (18). En efecto, a la hora de establecer similitudes, nuestra mente (específicamente, el funcionamiento psíquico resultante de la actividad del córtex prefrontal) recurre a asociaciones poco fidedignas desde la perspectiva de la objetividad y la lógica. Diríase que se conforma con coincidencias grosso modo. No entendemos aún bien el porqué de esta relativa indiscriminación perceptual y conceptual. En cierta manera, quizás 
tuviera razón David Hume, el filósofo escocés del siglo dieciocho, cuando dijo que, sencillamente, «las explicaciones son el lugar donde la mente busca reposo». Sea como fuere, esa tendencia que nos lleva a procurar encajar las nuevas percepciones en el recuerdo de otras más o menos parecidas tiene enorme importancia en la comprensión psicoanalítica de los conflictos psíquicos, porque es la base de lo que denominamos la transferencia.

La transferencia está constituida por el desplazamiento inconsciente hacia personas del presente implicadas en una relación interpersonal, de sentimientos, expectativas y reacciones experimentados originalmente en la niñez con figuras significativas. Los padres de la infancia internalizados suelen ser las figuras desde las que se desplazan estas pautas emocionales, pero también pueden ser fuente de éstas los hermanos, abuelos, vecinos, maestros o los héroes de nuestra niñez. Aunque se dan las distorsiones transferenciales en todo tipo de interacciones en la vida, es en la situación psicoanalítica donde se revelan con especial claridad e intensidad, porque el encuadre o setting psicoanalítico (el anonimato y la neutralidad del analista, y la regresión promovida por el uso del diván y el método de la libre asociación) está diseñado precisamente para ponerlas de manifiesto. La neurosis transferencial resultante (réplica de la neurosis infantil) se convierte entonces en objeto de exploración compartida entre psicoanalista y psicoanalizado. La finalidad del tratamiento es la resolución de dicha neurosis y la liberación de las potencialidades del paciente comprometidas por su patología. En palabras de la notable neuropsiquiatra y psicoanalista Regina Pally (19), «Una manera de definir el psicoanálisis es como el método de tratamiento que lleva al paciente a centrar su atención consciente en los detalles específicos de la situación transferencial con el fin de desarrollar una capacidad perceptual más ajustada a la realidad que le permita, si es necesario, generar categorías nuevas de experiencia interpersonal».

\section{Limitaciones del psicoanálisis}

Uno de los objetivos fundamentales del tratamiento psicoanalítico es el de conseguir un acceso consciente a las emociones patogénicas. Está empíricamente demostrado que la capacidad de poner en términos de concepto lingüístico el significado de las experiencias favorece la regulación de los afectos displacenteros, lo que recuerda aquello que escribiera Marcel Proust: "Cuando las penas se transforman en ideas pierden algo de su poder para herir nuestro corazón". Pero el intento de transformar los sentimientos en palabras topa indefectiblemente con obstáculos o resistencias (en la terminología psicoanalítica). Éstas resultan analizables en las psiconeurosis, pero no siempre en otras psicopatologías. Existe mucha experiencia acumulada respecto a que las características de ciertas enfermedades psiquiátricas pueden erigir una barrera infranqueable a este "trasvase" de sentimientos inconscientes a conceptos manejables racionalmente.

El asunto de la analizabilidad se ha debatido mucho en lo concerniente al fenómeno de la "pensée opératoire" (20) o alexitimia (21) (etimológicamente, 'incapacidad de leer los afectos'), trastorno descrito sobre todo en pacientes con enfermedades 
DEBATES

psicosomáticas. También ha existido gran controversia sobre las limitaciones del psicoanálisis en lo tocante a la psicoterapia de la esquizofrenia y a la influencia de las vivencias infantiles "esquizofrenogénicas". Se ha supuesto que durante los años formativos de la infancia, la cronicidad y la intensidad de ciertos mensajes contradictorios de "doble vínculo" (22) o la influencia de cierto tipo de "comunicaciones fragmentadas y amorfas" (23) podían hacer imposible esa integración de las autorrepresentaciones que normalmente resulta en la formación de un self cohesivo, desembocando esto entonces en cuadros clíni$\cos$ de psicosis. Este tipo de influencias adversas en la niñez producen, en efecto, psicopatología importante, pero se cree hoy día que ésta tenderá a ser caracterial, no esquizofrénica (24).

En estos casos y en otros caracteriales con un "Yo permanentemente unificado" (25) resulta imposible llevar a cabo un psicoanálisis clásico, porque para éste se requiere de la mente del paciente (o, más precisamente, de su Yo) una capacidad de escindirse sin descompensarse, en una parte que reviva las emociones en el registro de proceso primario del hemisferio cerebral derecho y otra que las conceptúe y verbalice en el lenguaje de proceso secundario propio del hemisferio izquierdo. Cuando resulta posible el psicoanálisis porque el trastorno del paciente no es de naturaleza psicótica o prepsicótica, el analista establece una "alianza terapéutica" (26) con el psiquismo racional de aquél que permite explorar con el método de la libre asociación (piedra angular de la técnica psicoanalítica) otras partes del psiquismo no racionales o paleológicas, conectando sentimientos con pensamientos y recuerdos con fantasías.

\section{Psicoanálisis y cientificismo}

Es común en círculos profesionales pensar que el psicoanálisis no puede proporcionar datos útiles para fines científicos $\mathrm{y}$, sin embargo, a través de sus aplicaciones clínicas y a pesar de sus limitaciones, el psicoanálisis permite el acceso a un material (inconsciente) que no puede obtenerse por ningún otro medio y sin el cual la comprensión del funcionamiento mental es incompleto (8).

El psicoanálisis clínico estudia y descifra de modo sistemático y de acuerdo con unas técnicas empíricamente establecidas, los significados implícitos en la producción verbal y paraverbal de los pacientes. Desde una perspectiva de filosofía de la ciencia y siguiendo a George Klein (27), malogrado investigador de Menninger, hay que señalar que $\left(1^{\circ}\right)$ las funciones psíquicas y sus significados son tan directamente observables y especificables como puedan serlo los tractos nerviosos o las descargas neuronales; $\left(2^{\circ}\right)$ las formulaciones sobre los significados discernibles en el material clínico constituyen una base explicativa tan adecuada como puedan serlo aquellas de naturaleza neurofisiológica; y $\left(3^{\circ}\right)$ estos significados definen unos principios de regularidad que pueden resultar traducibles, pero no reducibles, a los hallazgos neurobiológicos. Un mismo conjunto de fenómenos toma sentidos diferentes dependiendo del foco desde el que se interprete. Por ejemplo, un estado depresivo puede conceptuarse como secundario a una reacción de duelo 
patológico, pero también puede ser objeto de un sinnúmero de estudios de laboratorio. La primera interpretación de la situación no es menos "real" que la fisiológica cuantitativamente mensurable. Waelder (28) en su "principio de la función múltiple" formuló claramente la necesariedad de esta doble visión para la comprensión global de la mente humana. Se ha señalado (29) que esta formulación de Waelder es una versión psicoanalítica del principio de complementariedad de Niels Bohr, de 1927, que establece que toda ciencia ha de ser considerada desde dos perspectivas (experimental y de observación) mutuamente exclusivas -una analítica y otra sintética- para aspirar a representar un entendimiento adecuado de los fenómenos físicos (ejemplo: la simultánea naturaleza corpuscular y ondulatoria de los fotones).

No es raro encontrar en Psicología y Psiquiatría investigadores que piensan que la única función legítima del científico es ir en pos de los principios biológicos 0 , como mucho, reflexológicos que subyacen a las manifestaciones psicológicas complejas, suponiendo que solamente por medio del conocimiento de las leyes fisiológicas puede verdaderamente llegarse a conocer conductas que implican intencionalidad, significado y finalidad. Las formulaciones "mentalistas" psicoanalíticas no serían sino "meras reflexiones" basadas en los aspectos fisiológicos correspondientes (30). Los substratos neuroquímicos son iguales en toda la especie humana, pero ¿qué nos dice eso de la mente individual? Como señaló el famoso biólogo de Harvard, Stephen Jay Gould (31), "La bioquímica puede ser la misma, pero cuentan las circunstancias del contexto y el pasado". La realidad es que, por interesantes y precisos que sean los datos neurobiológicos, ni son de una categoría epistemológica mejor, ni nunca podrán sustituir a los datos de significado personal que constituyen el sector natural de estudio de la psicología psicoanalítica, porque pertenecen a órdenes de percepción completamente distintos. Se ha llegado a escribir que dentro de cien años no existirán más ciencias terapéuticas que la inmunología y el psicoanáli$\operatorname{sis}(32)$.

\section{Mente y cerebro}

Los fenómenos mentales no son asunto para decidir si el psiquismo posee o no base fisiológica. Naturalmente que la tiene. La intencionalidad, las motivaciones, los significados y cualquier otra manifestación psíquica normal o patológica, se hallan implementados neurofisiológicamente. En tiempos de Rudolf Virchow solía decirse que el cerebro segregaba pensamientos como el hígado bilis.

La mente es un epifenómeno del funcionamiento cerebral: una "propiedad emergente", que dicen los filósofos de la ciencia. Se define una propiedad emergente como aquella conectada causalmente con elementos o fenómenos anteriores, pero que no constituye una cualidad de ninguno de ellos ni resulta de su simple adición. Suele ponerse como ejemplo la liquidez del agua, formada por elementos no líquidos (el hidrógeno y el oxígeno). La actividad psíquica es consecuencia de la descarga de grupos neuronales de determinadas áreas del cerebro, pero la mente no equivale sencillamente al funcionamien- 
DEBATES

to de estas áreas cerebrales. Las secuencias causa-efecto no son sinónimo de identidad. El trueno sigue al relámpago, pero el trueno no es el relámpago.

La comprensión del sentido de la conducta y de las experiencias subjetivas se halla a un nivel de coherencia diferente de cualquier formulación de leyes fisiológicas. Dice George Klein (27), "Puesto que las intenciones son personales y relacionales, los modelos adecuados para la búsqueda de propósitos e intención no pueden ser formulados en términos de modelos impersonales [...] Realmente, la lectura de las intenciones o coherencias en las acciones y experiencias de otros no puede hacerse en los acontecimientos fisiológicos, al menos no más de lo que la finalidad del transporte puede ser colegida a partir del funcionamiento mecánico del automóvil". En el lenguaje filosófico, se trata de niveles distintos de discurso.

Michael Polanyi, químico, conocido filósofo de la ciencia y profesor de la Universidad de Manchester, escribió elocuentemente sobre lo necesario de incluir las experiencias y estados subjetivos dentro del campo del escrutinio científico. Polanyi (33) señaló: "Un neurofísico que observase los procesos que tienen lugar en la retina y en el cerebro de un hombre que está mirando algo no sería nunca capaz de interpretar a partir de los acontecimientos físicos bajo estudio qué está viendo el hombre en ese algo". El estudio de la percepción y la consciencia, en efecto, será siempre incompleto si no se añade al punto de vista objetivo el subjetivo. La comprensión global de estos fenómenos requiere, como dicen los psicolingüistas, las perspectivas de primera y tercera persona.

En el psicoanálisis precisamente se intenta comprender con la mayor objetividad posible el mundo subjetivo del analizado. Esta aparente contradicción significa que el material verbal del paciente que expresa su versión subjetiva del mundo es lo que se toma como objeto de estudio. Lo que se analiza no es la vida del paciente, sino su psiquismo (34). Lamentablemente, esta importante distinción no se tiene siempre presente en la práctica. Cualquier intento por teorizar cabalmente acerca de la mente consciente o inconsciente tendrá que saltar inferencialmente desde lo objetivamente constatable a lo interno subjetivo y viceversa. En nuestro estado actual de conocimientos muchos de estos saltos han de darse aún en el vacío, y es bien sabido que, como observaron los sabios de la Grecia clásica, nuestra naturaleza le tiene horror al vacío - a cualquier tipo de vacío. De aquí que, frecuentemente, en nuestras teorizaciones los psiquiatras hayamos tendido a usar los hallazgos objetivos que son cuantificables más como lugar al que aferrarnos que como plataforma desde la que saltar al estudio de la experiencia subjetiva (15).

\section{Multideterminación}

La acción humana está multideterminada, pero no por el cuerpo y el alma como se pensaba antiguamente, ni por el cerebro, por una parte, y la mente, por otra, como aún se piensa a veces, porque estas entidades no pueden estar contrapuestas. El organismo se halla multideterminado por lo genético y lo adquirido (nature and nurture). En el caso de la enfermedad depresiva, por ejemplo, existen componentes hereditarios de penetrancia 
variable, por una parte, y componentes adquiridos (neuróticos o psicóticos), internalizados en la niñez, por otra. Se han formulado sofisticados modelos psicobiológicos basados en extensos estudios genéticos, en los que se separan las dimensiones del temperamento (heredadas) de las del carácter, adquiridas y modeladas por las influencias familiares postnatales (35).

Otro tipo de multideterminación es el que encontramos en el modelo estructural ("segunda tópica") de Freud (16). En éste el funcionamiento mental se halla multideterminado por las instancias psíquicas Ello, Yo y Superyó. Estas son abstracciones teóricas que corresponden a conjuntos de motivación psíquica generalmente opuestos entre sí y cuyo sustrato neurofisiológico no tiene localización precisa (6). El Ello está compuesto por las pulsiones instintuales y otras tendencias internas compartidas con los animales. El Yo está al cargo de la evaluación racional de las realidades y de la actividad con fines adaptativos. El psicoanálisis, en efecto, ve al hombre tanto impelido por impulsos como guiado por propósitos. El Ello sería la continuación de lo que los biólogos conocen como las tendencias de dirección periférica de los organismos vivos, y el Yo, el representante del control central del organismo (28). Finalmente, en grado creciente desde una cierta edad del desarrollo infantil, existe el Superyó, el censor interno moral (o moralista) con sus exigencias y prohibiciones.

La multideterminación de la vida mental se halla ilustrada por la complejidad de los problemas a que ha de enfrentarse el Yo: algunos de éstos tienen claramente su origen en las circunstancias externas (conflictos extrapsíquicos) y otros resultan de la oposición a otras instancias internas (conflictos intrapsíquicos), esto es, del choque con la presión de los instintos, por una parte, y las exigencias ideales e imperativos categóricos, por otra. Cada problema general a que se enfrenta el Yo está formado por un grupo de estos elementos; por ejemplo, el problema de la gratificación instintual asignada al Ello contiene, naturalmente, tantos problemas como pulsiones hay en búsqueda de gratificación. Además, los conflictos intrapsíquicos no siempre son intersistémicos: dentro de una misma instancia psíquica también puede existir conflicto (intrasistémico), como sucede con la incompatibilidad entre algunas lealtades dentro del Superyó.

En su adaptación al mundo externo, el Yo ha de mediar entre las presiones del Ello y las exigencias del Superyó, pero el papel jugado por el Yo en la resolución de los problemas consiguientes no se limita al de una "centralita" pasiva. El Yo intenta dominar aloplásticamente -no sólo autoplásticamente (36)- el entorno y, en el desarrollo normal, muestra una actividad represora hacia la vida instintual, procurando incorporarla a su organización. Esta interacción ejemplifica por antonomasia el concepto psicoanalítico de la perspectiva dinámica, que resulta aplicable a cualquier acción humana (37). En efecto, nuestra vida psíquica parece estar regida por un principio de función múltiple (Waelder (28)), que dicta que no puede existir ninguna solución a un problema que no represente simultáneamente un intento de solución a otros problemas. Cada acto psíquico es consecuencia, en realidad, de un intento de dar solución simultánea a todos los problemas, si bien dichas soluciones siempre resultarán más satisfactorias para una instancia que para otra. Cualquier manifestación mental tendrá carácter de compromiso entre los instintos, 


\section{DEBATES}

las defensas opuestas a éstos y la adaptación a la realidad externa; y las transacciones a que llega el Yo podrán ser precarias y ansiógenas o relativamente saludables. Aun en el caso de estas últimas siempre existirá discordancia o tensión en algún sentido. Este reconocimiento puede proporcionarnos una clave para el entendimiento de la sensación de insatisfacción interna y contradicción perpetua que, en mayor o menor grado, compartimos todos los seres humanos.

\section{BIBLIOGRAFÍA}

(1) Shevrin, H. "Psychoanalytic and neuroscience research", The Amer. Psychoanalyst, 1998, 32 (3), p. 25.

(2) Freud, S. "La interpretación de los sueños”, 1900, O.C., Madrid, Biblioteca Nueva, 1973.

(3) Moore, B.E. y Fine, B.D. Términos y conceptos psicoanalíticos, 1990, Madrid, Biblioteca Nueva, 1997.

(4) Ellenberger, H.F. El descubrimiento del inconsciente, 1970, Madrid, Gredos, 1976.

(5) Freud, S. “Lo inconsciente”, 1915, O.C., Madrid, Biblioteca Nueva, 1973.

(6) Kline, P. Fact and Fantasy in Freudian Theory, Londres, Methuen, 1972.

(7) Fisher, S. Y Greenberg, R.P. The Scientific Credibility of Freud's Theories and Therapy, Nueva York, Basic Books, 1977.

(8) Paniagua, C. "Can clinical psychoanalysis be scientific?", Amer. J. Psychother., 1987, 41, pp. 104-116.

(9) Humphrey, N. Consciousness Regained, Oxford, Oxford Univ. Press, 1983.

(10) Rosenblatt, A.D. y Thickstun, J.T. “Modern Psychoanalytic Concepts in a General Psychology”, Psychol. Issues, Monogr. 11, Nueva York, Inter. Univ. Press, 1977.

(11) Pally, R. y Olds, D. "Consciousness: A neuroscience perspective", Int. J. Psychoanal., 1998, 79, pp. 971-989.

(12) Boorstin, D.J. Los creadores, 1992, Barcelona, Crítica, 1994.

(13) Pinker, S. How the Mind Works, Nueva York, W.W. Norton, 1997.

(14) Solms, M. "What is consciousness", J. Amer. Psychoanal. Assn., 1997, 45, pp. 681-703.

(15) Opatow, B. "The real unconscious: Psychoanalysis as a theory of consciousness", J. Amer. Psychoanal. Assn., 1997, 45, pp. 865-890.

(16) Freud, S. "El yo y el ello", 1923. O.C., Madrid, Biblioteca Nueva, 1973.

(17) Hobson, J.A. The Chemistry of Conscious States, Boston, Little \& Brown, 1994.

(18) en Schwaber, E.A. Interview. The Amer. Psychoanalyst, 1998, 32, pp. 27-28.

(19) Pally, R. "How the brain actively constructs perceptions", Int. J. Psychoanal., 1997, 78, pp. 587-593.

(20) Marty, P. y De M'Uzan, M. “La pensée opératoire”, Rev. Psychanalitique, 1963, supl., 27, pp. 345-356.

(21) Nemiah, J.C. y Sifneos, P.E. "Affect and fantasy in patients with psychosomatic disorders", en Hill, O.W. (ed.), Modern Trends in Psychosomatic Medicine, Londres, Butterworths, 1970.

(22) Bateson, G., Jackson, D.D., Haley, J. y Weakland, J.H. "Toward a theory of schizophrenia", Behav. Sci., 1956, 1, pp. 251-269.

(23) Wynne, L.C. y Singer, M.T. "Thought disorder and family relations in schizophrenics: Results and Implications", Arch Gen. Psychiat., 1965, 12, pp. 201-211. 
(24) Paniagua, C. "Las psicosis esquizofrénicas", en J.L.G. Rivera, A. Vela y J. Arana (eds.), Manual de Psiquiatria, Madrid, Karpos, 1980.

(25) Sterba, R. "The fate of the ego in analytic therapy", Int. J. Psychoanal., 1934, 15, pp. 117 126.

(26) Zetzel, E. “Current concepts of transference”, Int. J. Psychoanal., 1956, 37, pp. 369-376.

(27) Klein, G. Psychoanalytic Theory: An Exploration of Essentials, Nueva York, Int. Univ. Press, 1976.

(28) Waelder, R. "The principle of multiple function: Observations on overdetermination", 1930, Psychoanal. Q., 1936, 5, pp. 45-62.

(29) Frattaroli, E. "Antidepressants and psychotherapy: A false dichotomy?", 3 de diciembre, 1999, e-mail, @apsa.org.

(30) Kandel, E.R. "Biology and the future of psychoanalysis: A new intellectual framework for psychiatry revisited", Amer. J. Psychiat., 1999, 156, pp. 505-524.

(31) Gould, S.J. "Message from a mouse", TIME, 13 de septiembre, 1999, p. 60.

(32) Gunderson, J.G. y Gabbard, G.O. "Making the case for psychoanalytic therapies in the current psychiatric environment”, J. Amer. Psychoanal. Assn., 1999, 47, pp. 679-704.

(33) Polanyi, M. "The structure of consciousness", Brain, 1965, 88, pp. 799-810.

(34) Gray, P. El Yo y el análisis de la defensa, 1994, Madrid, Biblioteca Nueva, 1996.

(35) Cloninger, C.R., Svrakic, D.M. y Pryzbeck, T.R. "A psychobiological model of temperament and character", Arch. Gen. Psychiat., 1993, 50, pp. 975-990.

(36) Hartmann, H. La psicología del Yo y el problema de la adaptación, 1939, Buenos Aires, Paidós, 1987.

(37) Rapaport, D. y Gill, M.M. Aportaciones a la teoría y técnica psicoanalítica, 1959, Méjico, Pax, 1962.

*Diplomado de la Asociación Americana de Psiquiatría y Neurología

Miembro titular de la Asociación Psicoanalítica Americana

Corrrespondencia: C/. Corazón de María, 2 - 28002-MADRID

Fecha de recepción: 13-XII-1999 